\title{
Teaching Mindfulness-Based Stress Reduction (MBSR) Live Online to Individuals Suffering from Mental Fatigue - A Qualitative Evaluation
}

\author{
Birgitta Johansson $^{1} \&$ Helena Bjuhr ${ }^{1}$ \\ ${ }^{1}$ Department of Clinical Neuroscience and Rehabilitation, Institute of Neuroscience and Physiology, The Sahlgrenska \\ Academy, University of Gothenburg, Sweden \\ Correspondence: Birgitta Johansson, Department of Clinical Neuroscience and Rehabilitation, Institute of Neuroscience \\ and Physiology, The Sahlgrenska Academy, University of Gothenburg, Blå stråket 7, 41345 Gothenburg, Sweden
}

Received: January 27, 2016

Accepted: February 11, 2016

Available online: February 17, 2016

doi:10.11114/ijsss.v4i3.1389

URL: http://dx.doi.org/10.11114/ijsss.v4i3.1389

\begin{abstract}
An increasing number of studies report enhanced well-being for people with chronic diseases after attending an eight-week Mindfulness Based Stress Reduction (MBSR) program. The intention with this study was to qualitatively evaluate MBSR delivered live on the Internet for participants suffering from mental fatigue after an acquired brain injury (stroke or traumatic brain injury). The MBSR curriculum was strictly followed and was offered to participants in two groups live on the Internet $(n=20)$ and one face-to-face group $(n=12)$. Open questions were used to evaluate how participants managed to follow the program. Both the face-to-face and the live Internet MBSR groups reported very similar experiences after the program, such as increased awareness of the connection between body and mind, having more energy, experiencing greater clam, having more positive thoughts, and more hope and confidence for the future. Difficult experiences were connected to fatigability. It was primarily the weekly sessions that were strenuous. A sense of belonging to a group and sharing experiences with others was achieved in the live Internet groups as well as in the face-to-face group. In conclusion, the MBSR program can be delivered live online with positive effects for participants who suffer from mental fatigue after an acquired brain injury. However, for mentally fatigued participants, a slight adaption of the MBSR program is suggested.
\end{abstract}

Keywords: MBSR, live on Internet, qualitative, acquired brain injury

\section{Introduction}

\subsection{Mental fatigue after an acquired brain injury}

After traumatic brain injury (TBI) or stroke prolonged mental fatigue may occur and can be the main hindrance for participating in daily life with work and social activities (Belmont, Agar, Hugeron, Gallais, \& Azouvi, 2006; Staub \& Bogousslavsky, 2001). This post-injury mental fatigue is characterized by limited energy reserves and mental exhaustion becomes pronounced during sensory stimulation or when cognitive tasks are performed for extended periods without breaks. This can be difficult to understand as the fatigue may appear even after seemingly trivial mental activities, as reading a book or having a conversation with friends. Another typical feature is a disproportionally long recovery time needed to restore the mental energy after being exhausted. Common associated symptoms include: impaired memory and concentration capacity, slowness of thinking, irritability, tearfulness, sound and light sensitivity, sensitivity to stress, sleep problems, lack of initiative and headache (Johansson \& Rönnbäck, 2014b).

After the first year of neurological and neuropsychological recovery, mental fatigue can remain for many (Bushnik, Englander, \& Wright, 2008; Staub \& Bogousslavsky, 2001). The most important recommendations for alleviating mental fatigue include regular breaks, avoiding over-exertion, and doing one thing at a time. This is very hard to accept and it can take several years of considerable effort, frustration, despair and depression to find the right balance between activity and rest and to lead life in a new way.

\subsection{Mindfulness Based Stress Reduction (MBSR)}

The Mindfulness Based Stress Reduction (MBSR) program developed by John Kabat-Zinn (Kabat-Zinn, 2001) was considered an attractive treatment option for mental fatigue and co-occurring symptoms after a TBI or stroke. Several researchers have reported positive effects for chronic conditions including stress, depression, pain, and fatigue connected 
to cancer (L E Carlson \& S N Garland, 2005; Grossmana, Niemannb, Schmidtc, \& Walachc, 2004; Kabat-Zinn, Lipworth, \& Burney, 1985; Smith et al., 2008). We have also previously reported about reduced mental fatigue after an acquired brain injury (Johansson, Bjuhr, \& Rönnbäck, 2012). The intention with the MBSR program includes discovering the connection with mind and body, experiencing new perspectives, moving towards acceptance and compassion, relating to both oneself and to others (McCown, Reibel, \& Micozzi, 2011). These are considered important factors for people who suffer from mental fatigue, helping them to find new ways to deal with their difficulties in a more balanced and compassionate way.

Johansson et. al. (2012) found reduced mental fatigue and improved cognitive function (processing speed and attention) after the completion of an MBSR program for participants suffering from mental fatigue after TBI or stroke (Johansson et al., 2012). Other researchers also reported on positive effects after an MBSR course for people who had suffered a TBI with improved quality of life, perceived self-efficacy and cognition (Azulay, Smart, Mott, \& Cicerone, 2013), improved quality of life and decreased depression (Bedard and co-workers, 2003), and, after a stroke decreased depression (Joo, Lee, Chung, \& Shin, 2010). Fatigue was not included in these studies, but reduced fatigue after MBSR has been reported in other diseases e.g. for cancer patients (L E Carlson \& S N Garland, 2005), for people suffering from chronic fatigue syndrome (Surawy, Roberts, \& Silver, 2005) and having recourse to a mindfulness program closely following the MBSR program for multiple sclerosis participants (Grossman et al., 2010).

\subsection{Qualitative studies of mindfulness meditation}

Few qualitative studies of mindfulness meditation among clinical groups exist, but the individual experience of a mindfulness program can provide a deeper understanding of the positive and negative effects that mindfulness may have. A qualitative study with a slightly modeled MBSR program for older adults with chronic low back pain reported less pain, improved attention, improved sleep, enhanced well-being and improved quality of life (Morone, Lynch, Greco, Tindle, \& Weiner, 2008). Increased calm, enhanced sleep quality, greater energy levels, less physical pain and increased well-being (Kvillemo \& Bränström, 2011) and improved self-control, being able to respond in a more beneficial way and personal growth seeing the illness in a new perspective (Mackenzie, Carlson, Munoz, \& Speca, 2007) were reported after a MBSR program for patients with cancer.

\subsection{Mindfulness programs on the Internet}

The interest in mindfulness has increased, as well as the number of people who would like to attend a mindfulness program. However, it may be difficult for some to find a course close to where they are living or they may have functional restrictions which impede them from attending a mindfulness program. With the use of the Internet, more people are able to obtain access to mindfulness courses and it is today possible to attend mindfulness programs on the Internet. Research has been carried out on the positive effects of mindfulness delivered on the Internet for e.g. anxiety disorder (Boettcher et al., 2014), stress (Glück \& Maercker, 2011; Krusche, Cyhlarova, King, \& Williams, 2012; Morledge et al., 2013) and irritable bowel syndrome (Ljótsson et al., 2010). These studies were mindfulness interventions with a self-based program.

\subsection{The aim of the present study}

The purpose of this study was to describe the learning potential of an MBSR program delivered live on the Internet for participants suffering from mental fatigue after an acquired brain injury. The main themes and content of the program were securely kept, including the notion of belonging to a group. The qualitative analysis is based on open questions following the MBSR program.

\section{Method}

\subsection{Participants}

The participants included in the study were recruited from an advertisement in a local daily newspaper or from information published on our website www.mf.gu.se. We conducted an initial screening of potential participants via a telephone conversation. Selected and interested participants were later interviewed by a neurologist and a neuropsychologist to ascertain their acquired brain injury, co-morbidity and medication and were also evaluated to determine mental fatigue with the Mental Fatigue Scale (Johansson \& Rönnbäck, 2014a), depression and anxiety with the Comprehensive Psychopathological Rating Scale (Åsberg, Montgomery, Perris, Schalling, \& Sedvall, 1978) and cognitive ability (Johansson, Bjuhr, Karlsson, Karlsson, \& Rönnbäck, 2015). The inclusion criteria were as follows: men and women between 20 and 65 years of age; had been employed prior to suffering a stroke or traumatic brain injury (TBI); had recovered from neurological and neuropsychological symptoms and suffering from pathological mental fatigue for at least 6 months before inclusion in the study. Among those with a mild TBI, they had all been diagnosed acutely with a concussion. The exclusion criteria were as follows: psychiatric or neurological disorders; a history of alcohol or drug abuse; significant cognitive impairment; and having previously attended an MBSR program. 


\subsection{Research design}

It was not possible to accomplish the primary intention with a randomized study as we were not able to recruit enough participants in an area close to Gothenburg. We decided to run a feasibility study and allowed participants from the whole of Sweden to attend the Internet program. We also allowed seven participants to attend the MBSR program delivered face-to-face due to personal requests made by them. Either these participants did not want to attend an Internet program or they reported that using a computer for long periods was strenuous for them. However, age, $\mathrm{TBI} /$ stroke, gender, education, time since injury and working capacity did not differ between the groups (Table 1).

An active control group met once a week for eight sessions, for 1.5 hour of walking in the Gothenburg Botanical Garden. The active control group was later offered MBSR live online and their comments in response to the questions after MBSR are included in this analysis (Internet group 2). Figure 1 shows the study design with the groups included. In this study, we have merged the comments from both Internet groups to form one Internet group.

All participants provided an informed consent. The study was approved by the Ethical Review Board, Gothenburg, Sweden.

Table 1. Demographic data with mean values (standard deviation is included in brackets) and frequencies for those who completed the program. No significant differences between the background data were detected.

\begin{tabular}{llll}
\hline & MBSR face-to-face & MBSR Internet 1 & MBSR Internet 2 \\
\hline Numbers & 12 & 13 & 7 \\
Dropouts & 0 & 3 & 0 \\
Age & $48.0(9.4)$ & $46.3(11.5)$ & $47.6(8.9)$ \\
TBI/Stroke & $7 / 5$ & $6 / 7$ & $3 / 4$ \\
Gender (Females/Males) & $11 / 1$ & $11 / 2$ & $4 / 3$ \\
Education & 1 elementary & 1 elementary & \\
& 4 high school & 2 high school & 3 high school \\
& 7 university & 10 university & 4 university \\
Time since injury (months) & $120.5(152.0)$ & $76.2(75.5)$ & $53.8(53.9)$ \\
& range $11-513$ & range11-283 & range 8-168 \\
Working, \% of full-time (40 hrs./week) before brain & $100(0)$ & $92.3(19)$ & $100(0)$ \\
injury/stroke & & & $20.0(38)$ \\
Working, \% of full-time after & $29.2(38)$ & $46.1(47)$ & $21.6(5.4)$ \\
brain injury/stroke & & & $20.5(6.3)$ \\
MFS* baseline & $19.8(5.2)$ & & \\
\hline
\end{tabular}

MFS- Mental Fatigue Scale, a cutoff score of 10.5 (Johansson \& Rönnbäck, 2014a)

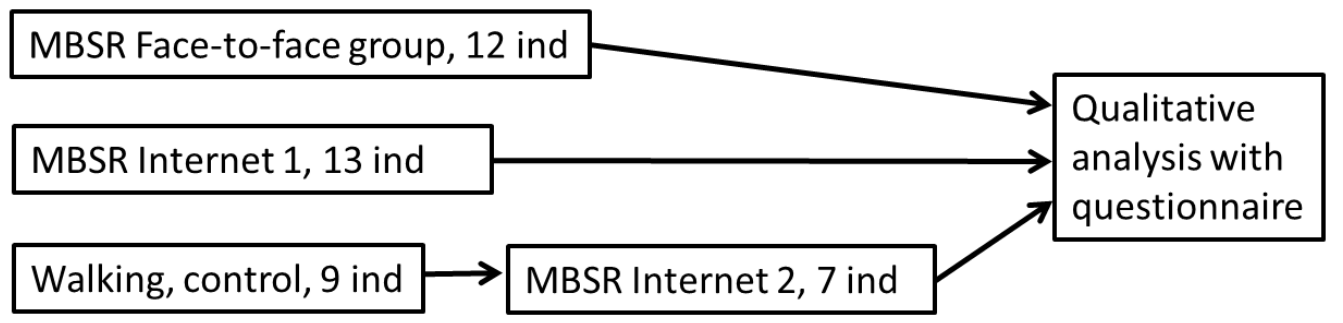

Figure 1. The study design.

\subsection{Procedure}

Mindfulness intervention involves exercises used in order to cultivate awareness of the present moment to become wise and compassionate, awake and aware in every-day life. The MBSR program we delivered online and to the face-to-face group followed the MBSR curriculum (Kabat-Zinn, 2001) with formal meditation, informal practices and group inquiries during the meetings. The formal practices include body scan to cultivate awareness of each area of the body; sitting meditation with an awareness of the breath and a systematic widening of the field of awareness to include all four foundations of mindfulness, namely awareness of the body, feeling tone, mental states and mental contents; and mindful hatha yoga. During the full-day session between sessions 6 and 7, walking meditation, loving-kindness 
meditation and mountain meditation were introduced. The participants were encouraged to practice at home six days/week. Home practice was supported by guided instructions on video recordings on the website and CDs. All participants were given CDs with body scanning (42 mins.), sitting meditation (10, 15 and 45 mins.), lying down yoga (48 mins.) and standing yoga (47 mins.). All participants also had access to a website for home practice with videos recorded for this course. The website included similar practices as the CDs, with the addition of walking meditation, and also instructions to guide them through the practices (Table 2). The participants also received written didactic material. They were also encouraged to introduce mindfulness in their daily lives with daily activities which they were encouraged to carry out mindfully with their full attention. The teaching intentions involved experiencing new possibilities for life satisfaction, experiencing physical sensations, sounds, thoughts and emotions as a whole and as the program proceeded, moving towards acceptance of life the way it is. Kindness and compassion skills were embedded in the entire program (McCown et al., 2011). The sessions for the two groups (face-to-face, Internet 1) were delivered during the same week, on two consecutive days. The Internet 2 program for the active control group was delivered directly after finishing the Internet 1 program. The mindfulness teacher, H. Bjuhr delivered the MBSR program. Dr. Bjuhr is a clinical psychologist and a certified MBSR teacher who was trained at the Center for Mindfulness, University of Massachusetts Medical School, USA.

\subsection{MBSR live on Internet}

The live Internet MBSR group used the Adobe Connect platform for online meetings. The participants logged in to the study website and were thereafter allowed to connect to the class. All participants used microphones and web cameras, allowing them to interact with the MBSR teacher. They were also able to establish contact with the other participants in the group. This enabled them to watch and listen to all the dialogues after the formal practices and it also enabled the Internet groups to experience a sense of belonging to a group, and to take part in the group inquiries, sharing experiences and learning from each other in an effort to increase the insight of mindfulness.

Table 2. Self-guided material for home practices accessible on the website developed for the purposes of the study.

\begin{tabular}{ll}
\hline Practice & Minutes \\
\cline { 2 - 2 } Introduction to mindfulness & 42 \\
Eating raisins & 22 \\
Reflection on eating raisins & 21 \\
Introduction to body scanning & 7 \\
Body scanning & 50 \\
Introduction to yoga & 18 \\
Instructions to yoga practices & 9 \\
Lying down yoga & 77 \\
Standing yoga & 60 \\
Introduction to sitting meditation & 16 \\
Instructions to sitting postures & 5 \\
Sitting meditation & 20 \\
Sitting meditation & 50 \\
Introduction to short breathing/breathing space & 7 \\
Short sitting breathing & 5 \\
Introduction to walking meditation & 19 \\
Walking meditation & 18 \\
\hline
\end{tabular}

\subsection{Data analysis}

After the completion of the MBSR courses, all the attendees answered a questionnaire. The intention was to evaluate the learning potential of an MBSR training program delivered live through the Internet-based from a perspective of mental fatigue. The questions included were about how the participants managed to follow the MBSR curriculum and which elements they experienced as being beneficial and which elements were difficult. The questions are shown in Table 3 . The data from this study are reported holistically. We wanted to show the diversity of individual experiences from the program as it can give valuable information and inspiration to others who are considering delivering an MBSR program. 
Table 3. Open Questions.

\begin{tabular}{ll}
\hline & Open questions in questionnaire \\
\hline 1 & How did you manage participating in the MBSR course? \\
2 & How did it work for you, learning the MBSR curriculum? \\
3 & What have you learned from the MBSR course? \\
4 & What did you gain from the MBSR course? \\
5 & Which elements have been difficult? \\
6 & How did you manage the full-day retreat (if you participated)? \\
7 & Have close relatives or friends noticed any change in you? \\
8 & Would you recommend this program to others who suffers from mental fatigue? \\
\hline
\end{tabular}

\section{Results}

During the MBSR program there were no drop-outs in the face-to-face group, three drop-outs after the first session in the Internet 1 group, and none in the Internet 2 group. Eleven of the 12 participants in the face-to-face group answered the questionnaire as did all 13 in the Internet group 1, and five of the seven participants from the Internet group 2.

\subsection{Questions 1-5, responses are overlapping and reported together.}

\subsubsection{Internet groups}

Most of the participants were satisfied with the internet course and with the methods of teaching the course. No participants were dissatisfied. Compared to the face-to-face group with no drop-outs, three dropped out after the first session. After finishing the course, the participants in the Internet courses reported less tension in the body, having become calmer and less worried. They also reported an improvement in sleep, a faster recovery time after being exhausted and having more energy during the day. They also reported relief from the sense of loss for the activities that they were able to do before the injury. Many participants could also manage noise better, having an improved focus on work, and an improved focus on reading. Increased happiness was also reported as well as a greater acceptance and an ability to relate to themselves with greater compassion, more patience and hope for the future and having learned to stop and reflect in their busy lives and to "listen to myself more closely". Some also became better at adapting to mental fatigue and realized how energy-demanding many activities are and they learned to do less as "I don't need to do everything". Feelings of inner safety and greater contentment with life were also expressed. The didactic material was reported as being inspiring and a help for self-reflection and insight, while reading the text was also difficult and strenuous for others. Talking in turn during the inquiries was a beneficial and energy-saving strategy.

A few were struggling with their technical equipment, and become stressed, especially during the first sessions, but they learned how to handle the equipment and were able to handle it more smoothly later on. They had all checked their connections with their microphones and web cameras before the start of the course. As participants varied in computer experience and also fatigue, some needed more support than others. The sound during the dialogue was occasionally experienced as disturbing due to differences in personal equipment including headsets, computers or Internet connections. However, sensitivity to noise was decreased significantly for Internet participants (Wilcoxon signed ranked test, $\mathrm{p}=0.005$, Sensitivity to noise, item from the Mental Fatigue Scale used in our quantitative analysis (Johansson et al., 2015).

Several participants made comments about the length of the inquiries after the weekly practice, as this was long and strenuous. On the other hand, many wanted time for questions and for hearing the responses from the teacher, this being reported as an important part of the sessions. One participant expressed a wish to have direct contact with the teacher due to thoughts and feelings which the participant experienced during the practices.

\subsubsection{Face-to-face group}

Eleven $(85 \%)$ of the participants were very satisfied with the course including the teaching method and had gained positive experiences. A feeling of safety was reported and they appreciated belonging to a group and sharing experiences with others. They had started to carry out daily activities in a more relaxed way, having more positive thoughts, and being more aware about the connection with body and mind and inner states such as thoughts, emotions and physical sensations. They had increased their inner calm. The participants reported that the mindfulness practices brought hope and confidence for the future, as well as a curiosity for the body, the participants becoming more attuned to what is going on in their bodies. They reported having learned to breathe and they reported that they had learned the meditation techniques and the technique of taking one or more rest periods during the day and adapting better to personal needs with "greater self-forgiveness, and the notion that all must not be done at such a high speed". The self-guided meditations 
for home practices on the website and CDs were helpful.

However, fatigue was an evident hindrance, and after the 2.5-hour weekly sessions, increased fatigue was reported for many participants. The written didactic material was, for some hard to use as they experienced difficulties reading due to fatigue. Some participants felt that there was too much home-work to do between the sessions. This resulted in a feeling of being stressed. One participant was dissatisfied with the face-to-face MSBR intervention, having not gained anything from the program nor from the curriculum which the participant was skeptical about, and one participant was partially satisfied with the course.

\subsubsection{Common experiences reported by both groups}

Prioritizing time for home practice was a common challenge. The face-to-face group and the Internet groups 1 and 2 did not differ in time spent on home practice. They practiced on average between 27 and 30 mins./day. However, there was a vast variation between not having practiced at all, and having practiced 52 minutes/day. Sixty-eight per cent had practiced at home four times/week or more. Some managed to include both formal and informal practices. Others included more of the informal practice and had difficulties finding the time and space for formal meditation practice. Restlessness during meditation was reported by several, while others appreciated the time involved in the practices and they felt calm during the meditation. For those experiencing pain, sitting and yoga practices were reported as being difficult, while others, despite the pain could find a suitable position to be in. However, they were all informed that they were allowed to move from one position to another, and, during the yoga practice, to adjust their practice to suit their abilities or to perform the yoga practices in the mind.

\subsection{Question 6: the full-day retreat}

\subsubsection{Internet}

Sixty-two per cent from the Internet 1 group and $71 \%$ from the Internet 2 group participated in the full-day retreat. All participants reported that they had gained positive experiences from the retreat and a greater insight into mindfulness. They also expressed that the day was a wonderful experience with the following comments: "less strenuous than expected", "thought it would be strenuous, but it turned out to be the contrary", "appreciated being quiet for a whole day" the day was "better than expected, the "sense of time disappeared, "managed to perform all the meditations" and "the day passed quickly". Increased fatigue after the full-day retreat was reported by some.

\subsubsection{Face-to-face group}

Those who participated ( $83 \%$ ) reported positive experiences from the full-day retreat, and expressed that the day was wonderful, "fantastic experience", the day "opened doors", and they also appreciated performing the practices together with others, having a sense of belonging, even if they did not talk to, or have eye contact with each other, "cozy atmosphere and we made it together, happy for this". However, many also reported an increased tiredness the following day and also for several days after the retreat. "I want more, but I was tired afterwards".

\subsection{Question 7: have relatives or friends noticed any changes in you?}

\subsubsection{Internet group}

Seventy percent of the participants reported comments from relatives and friends as they were less tired, happier, calmer, less irritated and had more patience.

\subsubsection{Face-to-face group}

Thirty percent of the participants reported relatives or friends having seen them as calmer and happier people and one as being more tired (was not satisfied with the course).

3.4 Question 8: would you recommend the program?

\subsubsection{Internet}

All would recommend MBSR for others with mental fatigue and one suggested it to be implemented in the rehabilitation clinics.

\subsubsection{Face-to-face group}

Eleven ( $85 \%$ ) of the participants would recommend the MBSR program to others with mental fatigue. One had doubts about the program and one would not recommend it.

\section{Discussion}

People suffering from mental fatigue after an acquired brain injury (stroke or traumatic brain injury) have reported decreased mental fatigue after an MBSR program (Johansson et al., 2012). However, as many of the participants live far 
away from the center where an MBSR program is delivered or may have functional restrictions impeding them from attending a face-to-face MBSR program, we wanted to study whether it is possible to deliver the MBSR program live on the Internet. The intention was to obtain information concerning learning potential and to find out how the program was experienced by participants suffering from mental fatigue after an acquired brain injury. In this study we evaluated, by means of qualitative interviews, with open questions, the MBSR program delivered live on the Internet, and compared it with the outcome for a face-to-face MBSR group.

\subsection{Reported positive effects}

The reported answers showed the possibility to deliver MBSR live online for mental fatigue participants and also to obtain positive effects, similar to those for a face-to-face MBSR group. Many of the participants, both in the Internet group and the face-to-face group reported reduced mental fatigue, greater calm, more satisfaction with life and they had gained more hope and confidence for the future. Happiness and greater acceptance were also expressed. In addition, the quantitative analysis showed significantly reduced mental fatigue for the Internet MBSR group and also improved attention (ability to process two temporally close targets) (Johansson et al., 2015).

Despite being easily exhausted, the full-day retreat was a positive experience for most of those who participated. It was also possible to deliver the full day live on the Internet obtaining a similar experience among the participants as in the face-to-face group. The daylong retreat could be a challenge for those suffering from mental fatigue. However, the day gave the attendees new insight into activities that they could cope with and benefit from by using new approaches. One important element was the calmness sustained for a longer period and also the absence of communication between participants during the day (no talking and no eye-contact). Some experienced greater calm and less fatigue after the day, while others experienced greater fatigue.

\subsection{Hindrances for participation}

The hindrances for the practice of mindfulness in the Buddhist tradition are desire, aversion, tiredness, sloth and torpor, restlessness and doubt (Goldstein \& Kornfield, 1987). These hindrances are referred to in this study. When suffering from mental fatigue, the hindrances become even more pronounced due to loss of energy. The right amount of energy is considered most beneficial to enable mindfulness training. Falling asleep during a body scan, especially during the first sessions was common, but the ability to stay awake increased during the course. Several participants from both the face-to-face and the live Internet MBSR programs reported similar difficulties, being fatigued during the sessions and after the sessions. The length of the sessions and especially the length of the inquiry during the weekly sessions were most strenuous, but the dialogue was also highly appreciated. The participants had a lot of questions to ask about their practices and many wanted feedback from the teacher. However, communication is, for people suffering from mental fatigue after an acquired brain injury extremely energy-demanding. The online course only allowed one person at a time to talk and this was reported as being beneficial. The participants were able to rest and just listen during the inquiry while waiting for their turn. The use of the dialogues to practice balance in energy during communication could be a way of working with fatigability. The special dialogue technique, the Insight dialogue, which involves listening and talking with more time and space and not being interrupted by others, could be beneficial (Kramer, 2007).

Several reported difficulties getting started with the home practices. This may be even harder for those participants suffering from fatigue as taking initiative is a common symptom belonging to the cluster of mental fatigue symptoms (Johansson \& Rönnbäck, 2014b). To start with shorter meditations can be beneficial if 45-minute meditation is too demanding. A program lasting for more than eight weeks can also give people with fatigue a better opportunity to establish new habits and to be better prepared to continue with the meditations after the program.

Another problem for some participants was that they had difficulties reading the didactic material, and this is also a common problem when suffering from mental fatigue, as reading takes a lot of energy and they also read at a slower pace (Johansson, Berglund, \& Rönnbäck, 2009). In order to overcome this problem, the text could be pre-recorded on audio-files.

Noise was also reported as disturbing for many in both groups. Sensitivity to noise i.e. being more easily disturbed and also in terms of experiencing higher sound volumes than normal is a common symptom associated with mental fatigue (Johansson, Starmark, Berglund, Rödholm, \& Rönnbäck, 2010). However, during the course, participants learned to handle noise better and also reported being less sensitive to noise after the MBSR program.

Desire, or the "if only" mentality was recognized among participants who have struggled for a long time with their mental fatigue. These participants were grasping for ways to become less fatigued, having a strong desire to change their life and find relief. A comment from one person who became aware of this desire, reported at the end of the course that "Now I know, there is no quick fix for mental fatigue". This is a great start, to obtain an insight into the difficulties, albeit one that is hard to overcome. However, this acceptance is a prerequisite for managing life better. 


\subsection{The group intervention}

A group setting is thought to be an important part of the MBSR intervention, not only for the social support, but also as a means of learning from the inquiry between the teacher and the participants. This learning is important for the formal and informal practices and for implementing mindfulness in daily life (McCown et al., 2011). The sense of belonging to a group and sharing experiences with others was also expressed as being beneficial for the majority in this study. The MBSR live on the Internet gave the participants, in the same way as the face-to-face group, a sense of belonging to a group, and the opportunity to share experiences with others and to receive immediate feedback from the teacher. The use of web cameras and microphones enabled the participants and also the teacher to watch and listen to each other during each session, creating a feeling of closeness to each other.

\subsection{Limitations}

This was a study with few participants and the study was not randomized. However, the subjective reports from participants in this study can give valuable information about the possibility of delivering the MBSR program live online and also the opportunity to deliver the demanding MBSR program to a group of participants who suffer from fatigue, which is one of the hindrances for practicing meditation.

\subsection{Conclusion}

In conclusion, this qualitative evaluation shows that the MBSR program delivered live on the Internet can be addressed to mental fatigue participants with positive effects which are important for well-being. The sense of belonging to a group, and sharing experiences with others were also possible to achieve with the live Internet groups. The program could, with slight adaptions to the sessions, suit fatigue participants more readily, with a slower pace, less talking, more repetitions and inquiries adapted in accordance with their mental energy.

\section{Acknowledgments}

This work was supported by grants from The Health \& Medical Care Committee of the Västra Götaland Region, The Swedish Stroke Association, and The Swedish Association for Survivors of Accident and Injury.

\section{References}

Azulay, J., Smart, C. M., Mott, T., \& Cicerone, K. D. (2013). A pilot study examining the effect of mindfulness-based stress reduction on symptoms of chronic mild traumatic brain injury/postconcussive syndrome. Journal of Head Trauma Rehabilitation, 28(4), 323-331.

Belmont, A., Agar, N., Hugeron, C., Gallais, B., \& Azouvi, P. (2006). Fatigue and traumatic brain injury. Annales de readaptation et de medecine physique, 49, 283-288.

Boettcher, J., Åström, V., Påhlsson, D., Schenström, O., Andersson, G., \& Carlbring, P. (2014). Internet-Based Mindfulness Treatment for Anxiety Disorders: A Randomized Controlled Trial Behavior Theraphy, 45, 241-253.

Bushnik, T., Englander, J., \& Wright, J. (2008). Patterns of fatigue and its correlates over the first 2 years after traumatic brain injury. Journal of Head Trauma Rehabilitation, 23(1), 25-32.

Carlson, L. E., \& Garland, S. N. (2005). Impact of mindfulness-based stress reduction (MBSR) on sleep, mood, stress and fatigue symptoms in cancer outpatients International Journal of Behavioral Medicine, 12(4), 278-285.

Carlson, L. E., \& Garland, S. N. (2005). Impact of mindfulness-based stress reduction (MBSR) on sleep, mood, stress and fatigue symptoms in cancer outpatients. International Journal of Behavioral Medicine, 12(4), 278-285.

Glück, T. M., \& Maercker, A. (2011). A randomized controlled pilot study of a brief web-based mindfulness training. BioMedCentral Psychiatry, 11(175), 1-12. http//dx.doi.org/10.1186/1471-244X-11-175

Goldstein, J., \& Kornfield, J. (1987). Seeking the Heart of Wisdom: The Path of Insight Meditation. Boston: Shambala Publications, INC.

Grossman, P., Kappos, L., Gensicke, H., D'Souza, M., Mohr, D. C., Penner, I. K., \& Steiner, C. (2010). MS quality of life, depression, and fatigue improve after mindfulness training: a randomized trial. Neurology, 75(13), 1141-1149.

Grossmana, P., Niemannb, L., Schmidtc, S., \& Walachc, H. (2004). Mindfulness-based stress reduction and health benefits: A meta-analysis. Journal of Psychosomatic Research 57 (2004) 35-43, 57, 35-43.

Johansson, B., Berglund, P., \& Rönnbäck, L. (2009). Mental fatigue and impaired information processing after mild and moderate traumatic brain injury. Brain Injury, 23(13-14), 1027-1040.

Johansson, B., Bjuhr, H., Karlsson, M., Karlsson, J-O., \& Rönnbäck, L. (2015). Mindfulness-Based Stress Reduction (MBSR) Delivered Live on the Internet to Individuals Suffering from Mental Fatigue After an Acquired Brain Injury. Mindfulness, 6, 1356-1365. 
Johansson, B., Bjuhr, H., \& Rönnbäck, L. (2012). Mindfulness based stress reduction improves long-term mental fatigue after stroke or traumatic brain injury. Brain Injury, 26(13-14), 1621-1628.

Johansson, B., \& Rönnbäck, L. (2014a). Evaluation of the Mental Fatigue Scale and its relation to Cognitive and Emotional Functioning after Traumatic Brain Injury or Stroke. International Journal of Physical Medicine and Rehabilitation, 2, 182. http//dx.doi.org/10.4172/2329-9096.1000182

Johansson, B., \& Rönnbäck, L. (2014b). Long-Lasting Mental Fatigue After Traumatic Brain Injury - A Major Problem Most Often Neglected Diagnostic Criteria, Assessment, Relation to Emotional and Cognitive Problems, Cellular Background, and Aspects on Treatment. In F Sadaka (Ed.), Traumatic Brain Injur. Rijeka, Croatia: INTECH. http//dx.doi.org/10.5772/57311

Johansson, B., Starmark, A., Berglund, P., Rödholm, M., \& Rönnbäck, L. (2010). A self-assessment questionnaire for mental fatigue and related symptoms after neurological disorders and injuries. Brain Injury, 24(1), 2-12.

Joo, 1H M., Lee, S. J., Chung, Y. G., \& Shin, Y. (2010). Effects of Mindfulness Based Stress Reduction Program on Depression, Anxiety and Stress in Patients with Aneurysmal Subarachnoid Hemorrhage. J Korean Neurosurg Soc, 47(5), 345-351.

Kabat-Zinn, J. (2001). Full Full catastrophe living: How to cope with stress, pain and illness using mindfulness meditation. London, 15th ed.: Piatkus Books.

Kabat-Zinn, J, Lipworth, L., \& Burney, R. (1985). The clinical use of mindfulness meditation for the self-regulation of chronic pain Journal of Behavioral Medicine, 8(2), 163-190.

Kramer, G. (2007). Insight Dialogue: The Interpersonal Path to Freedom. Boston: Shambhala Publications.

Krusche, A., Cyhlarova, E., King, S., \& Williams, J. M. G. (2012). Mindfulness online: a preliminary evaluation of the feasibility of a web-based mindfulness course and the impact on stress. BioMedJournal Open. http//dx.doi.org/10.1136/bmjopen-2011-000803

Kvillemo, P., \& Bränström, R. (2011). Experiences of a mindfulness-based stress-reduction intervention among patients with cancer. Cancer Nurs, 34(1), 24-31.

Ljótsson, B., Falk, L., Wibron Vesterlund, A., Hedman, E., Lindfors, P., Rück, C.,\& Andersson, G. (2010). Internet-delivered exposure and mindfulness based therapy for irritable bowel syndrome - A randomized controlled trial. Behaviour Research and Therapy, 48(6), 531-539.

Mackenzie, M. J., Carlson, L. E., Munoz, M., \& Speca, M. (2007). A qualitative study of self-perceived effects of mindfulness-based stress reduction (MBSR) in a psychosocial oncology setting. Stress and Health, 23(1), 59-69.

McCown, D., Reibel, D., \& Micozzi, M. S. (2011). Teaching mindfulness. A practical guide for clinicians and educators. New York: Springer.

Morledge, T. J., Allexandre, D., Fox, E., Fu, A. Z., Higashi, M. K., Kruzikas, D. T., . . Reese, P. R. (2013). Feasibility of an Online Mindfulness Program for Stress Management-A Randomized, Controlled Trial. Annals of Behavioral Medicine. http//dx.doi.org/10.1007/s12160-013-9490-x

Morone, N. E., Lynch, C. S., Greco, C. M., Tindle, H. A., \& Weiner, D. K. (2008). "I felt like a new person." the effects of mindfulness meditation on older adults with chronic pain: qualitative narrative analysis of diary entries. J Pain, 9(9), 841-848.

Smith, B W, Shelley, B M, Dalen, J, Wiggins, K, Tooley, E, \& Bernard, J S. (2008). A pilot study comparing the effects of mindfulness-based and cognitive-behavioral stress reduction. J Altern Complement Med, 14(3), 251-258.

Staub, F., \& Bogousslavsky, J. (2001). Fatigue after stroke: A major but neglected issue. Cerebrovascular Diseases 12, 75-81.

Surawy, C., Roberts, J., \& Silver, A. (2005). The Effect of Mindfulness Training on Mood and Measures of Fatigue, Activity, and Quality of Life in Patients with Chronic Fatigue Syndrome on a Hospital Waiting List: A Series of Exploratory Studies. Behavioural and Cognitive Psychotherapy, 33(1), 103-109.

Åsberg, M., Montgomery, S. A., Perris, C., Schalling, D., \& Sedvall, G. (1978). A comprehensive psychopathological rating scale. Acta Psychiatr Scand 271(suppl), 5-27.

\section{$(\mathrm{cc}) \mathrm{EY}$}

This work is licensed under a Creative Commons Attribution 3.0 License. 